\title{
On Dirichlet problems for some degenerate elliptic equations
}

$\mathrm{By}$

\section{Akira NAKAOKA}

(Received March 27, 1970)

1. Introduction. Recently the boundary value problems for degenerate elliptic equations have been studied by many authors. In treating these problems, at first we encounter the difficulty to give a unified formulation as compared with the regular (nondegenerate) cases. For example, consider the following two equations in $R_{+}^{1}$ :

$$
\begin{aligned}
& -\rho(x) \frac{d^{2} u}{d x^{2}}+u=f \\
& -\frac{d}{d x}\left(\rho \frac{d u}{d x}\right)+u=f
\end{aligned}
$$

where $\rho(x)$ is a non-negative bounded function vanishing only at $x=0$. If we give $f(x)$ in $L^{2}\left(R_{+}^{1}\right)$ to both equations, we shall have to seek a solution in different function spaces when we want to seek it in a subspace of $L^{2}\left(R_{+}^{1}\right)$. In fact, when we seek the variational solution of (1.2), it is natural to seek a solution $u(x)$ such that $u$ and $v \bar{\rho} \frac{d u}{d x}$ belong to $L^{2}\left(R_{+}^{1}\right)$, and moreover (as a result) $-\frac{d}{d x}\left(\rho \frac{d u}{d x}\right) \in L^{2}\left(R_{+}^{1}\right) \quad$ (for (1. 1), see [5]).

Now let $\rho(x)=\sqrt{x}$ and $u(x)$ be a function to be equal to $\sqrt{x}$ near $x=0$ and of bounded support, then $u, v \bar{\rho} \frac{d u}{d x}$ and $-\frac{d}{d x}\left(\rho \frac{d u}{d x}\right)$ all belong to $L^{2}\left(R_{+}^{1}\right)$, but $-\rho \frac{d^{2} u}{d x^{2}}$, which behaves like $x^{-1}$ near $x=0$, is not in $L^{2}\left(R_{+}^{1}\right)$. This suggests that it is not suitable to treat 
(1.1) and (1.2) in the same function space. Thus it will be necessary to introduce some special function spaces attached to the concerning equations in which the arguments can be developed.

M. S. Baouendi $[1]$, in his thèse, treated an operator such that

$$
A(x, D)=\Lambda^{*} \Lambda+\varphi(x)^{\rho} B(x, D) \phi(x)^{\mathrm{\rho}} \quad(\rho: \text { positive integer })
$$

where $\Lambda$ is a transversal differential operator of first order, $\varphi(x)$ is a smooth function vanishing on the boundary $(d \varphi \neq 0$ on the boundary) and $B(x, D)$ is an elliptic operator of second order. His operator degenerates only for the tangential direction. M.K.V. Murthy-G. Stampucchia [3] considered an operator of the form

$$
A(x, D) u=-\sum_{j, k=1}^{n} \frac{\partial}{\partial x_{j}}\left(a_{j k}(x) \frac{\partial u}{\partial x_{k}}+d_{j}(x) u\right)+\sum_{j=1}^{n} b_{j}(x) \frac{\partial u}{\partial x_{j}}+c(x) u
$$

such that $\sum_{j, k=1}^{n} a_{j k}(x) \xi_{j} \xi_{k} \geq m(x)|\xi|^{2}$ for $\vee \xi \in R^{n}$, where $m(x)$ a nonnegative function with $m(x)^{-1} \in L^{t}(\Omega)$ and they obtain the variational solution in some special function (weighted Sobolev) space. And N. Shimakura [7] has given a non-variational formulation of the boundary value problems for some degenerate elliptic equations in a half-space. In his localized problem the degenerate weight functions are restricted to the polynomial in the normal variable and his method is related with the theory of analytic functions.

In this paper we shall mainly concern with the following operator $A(x, D)$ of order 2 in a smooth domain $\Omega$ in $R^{n}$, which can be expressed in each sufficiently small boundary patch as

$$
A(x, D)=-\sum_{j, k=0}^{n-1} \tilde{a}_{j k}(x) \partial_{j} \partial_{k}+\text { first order operator, with }
$$

$\sum_{j, k=0}^{n} \tilde{a}_{j k}(x) \geqq c \sum_{j=0}^{n-1} \xi_{j}^{2}\left(c>0, \tilde{a}_{j k}=\overline{a_{k j}}\right)$. where $\partial_{0}=\rho(r) \frac{\partial(\xi)}{\partial \nu}$ and $\partial_{j}=\frac{\partial}{\partial \tau_{j}}$

$\Leftrightarrow \frac{\partial}{\partial \nu}$ and $\frac{\partial}{\partial \tau_{j}}(j=1, \cdots, n-1)$ are defined as follows: take a point $x_{0} \in \Gamma$, and introduce a curvilinear coordinate system $\sigma(\Gamma)$ on $\Gamma$ with its origin $x_{0}$. Since $\Gamma$ is smooth, if we take a sufficiently small neighbourhood $V\left(x_{0}\right)$ of $x_{0}$ we can find for any $x \in V\left(x_{0}\right)$ one and only one point $y \in \Gamma$ such that the vector $\vec{y} x$ coincides with the normal at $y$. Next let us denote $\Gamma,=\left\{x \in \mathrm{V}\left(x_{0}\right) ; \operatorname{dis}(x, \Gamma)\right.$ along the normal $<r\}$. We understand that on $\Gamma$ there is defined the curvilinear coordinate system $o\left(\Gamma_{r}\right)$ induced by that on $\Gamma\left(=\Gamma_{0}\right)$ for each $r>0$, and we define the local coordinate $\left(\nu, \tau, \cdots, \tau_{x-1}\right)$ of $x \in \mathrm{V}\left(x_{0}\right) \cap \Gamma_{r}(r \geqq 0)$ by (Continue to p. 377.) 
$(j=1, \cdots, n-1)$, which we say the weighted normal differentiation and the tangential differentiations respectively, $r(x)$ denotes the distance from $x \in \Omega$ to $\Gamma$, the boundary of $\Omega$, along the normal, and $0<\alpha<1$ and $\rho(r)$ will be given in section 2 .

Thus our operator degenerates only for the normal direction.

Our main aim is to determine the admissible lower order terms for Dirichlet problems and to establish the existence theorem of the solution in some weighted Sobolev space which will be defined in section 3 , (i. e) we don't use the usual $L^{2}(\Omega)$ as a basic space, but we use a kind of weighted $L^{2}(\Omega)$-space with its norm

$$
\|u\|_{0, r^{\infty}}^{2}=\int_{0}|u|^{2} \frac{d x}{r^{\infty}}
$$

which is stronger than the usual one.

Section 2 is devoted to the study of the weighted Sobolev spaces in a half-space, which is the preparation to section 3 .

In section 4 we shall obtain a weak solution using the Gàrding inequality in the weighted Sobolev space and using the Lax-Milgram lemma, and we shall show in section 5 its differentiability. In the final section we shall slightly make mention of the Dirichlet problems for higher order cases.

The author should like to express his sincere thanks to Professor S. Mizohata for the many valuable comments, for the

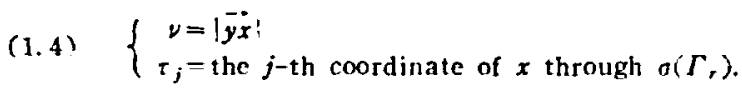

Thus we define

$$
\left\{\begin{array}{l}
\frac{\partial}{\partial \nu}=\sum_{k=1}^{n} \frac{\partial x_{k}}{\partial \nu} \frac{\partial}{\partial x_{k}} \\
\frac{\partial}{\partial \tau_{j}}=\sum_{k=1}^{n} \frac{\partial x_{k}}{\partial r_{j}} \frac{\partial}{\partial x_{k}}
\end{array}\right.
$$

We can see that the condition

$$
\sum_{j, k=0}^{1} a_{j k} \dot{\xi}_{j} \xi_{k} \geq c \sum_{j=0}^{1} \xi_{j}^{2}
$$

is invariant to the choice of local coorcinates $\sigma(\Gamma)$. Moreover, denoting $e_{0}=\left(\frac{\partial x_{1}}{\partial \nu}, \cdots, \frac{\partial x_{n}}{\partial \nu}\right), \vec{e}_{j}=\left(\frac{\partial x_{1}}{\partial \tau_{i}}, \cdots, \frac{\partial x_{n}}{\partial \tau_{j}}\right)$, we see that $\left|\vec{e}_{0}\right|=1$, and $\left(\vec{e}_{0}, \vec{e}_{j}\right)=0(j=1$, $\cdots, n-1)$, and the Jacobian $J\left(=\operatorname{det}\left(e_{0}, e_{1}, \cdots, e_{n},\right)\right)$ is a function of $\left(\tau_{1}, \cdots, \tau_{n-1}\right)$. 
constructive critisism of the manuscript and the constant encouragement.

2. Sobolev spaces with weights in $R_{+}^{n}$. In this section we introduce the Sobolev spaces with weights in which we develop our arguments. Let $\rho(x)$ be a real valued function such that

i) $\rho(x) \in C^{\infty}\left(R_{+}^{1}\right) \cap C^{0}\left(\bar{R}_{+}^{1}\right)$

ii) $0 \leq \rho(x) \leq M$ and $\rho(x)$ never vanishes except at $x=0$

iii) $\quad c_{1} x^{\alpha} \leq \rho(x) \leq c_{2} x^{\alpha}$ and $\left|\rho^{(m)}(x)\right| \leq c_{m} x^{\alpha-m} \quad(m=1,2, \cdots)$ when $0<x<a \quad(a$; a positive number and $0<\alpha<1)$.

iv) $\rho(x) \geqq \gamma>0$ and $\left|\rho^{(m)}(x)\right| \leq K_{m} \quad(m=1,2, \cdots)$ when $x \rightarrow \infty$.

We extend $\rho(x)$ to $R^{1}$ by $\rho(-x)$ for negative $x$ and denote the extended function by $\tilde{p}(x)$ and introduce $\phi(x)$ and $\tilde{\phi}(x)$ by setting

$$
\phi(x)=\int_{11}^{x} \frac{d \xi}{\rho(\xi)}
$$

$$
\widetilde{\phi}(x)=\int_{0}^{x} \frac{d \xi}{\tilde{\rho}(\xi)} .
$$

Now let us define our weighted Sobolev spaces by

Definition 2.1. A distribution $u(x, y)$ on $R_{+}^{n}=\{(x, y) ; x>0$, $\left.y \in R^{n-1}\right\}$ is said to be in $W^{m}\left(R_{+}^{n}, \phi\right)$ if and only if

$$
\|u\|_{m, \phi}^{2}=\sum_{j, \mid: \backslash \leq m} \int_{R_{+}^{n}}\left|D_{p}^{j} D_{v}^{k} u\right|^{2} d \phi d y
$$

is finite, where $D_{\rho}$ means $-i \rho(x) \frac{\partial}{\partial x}$ and $D_{\nu}^{k}=(-i)^{|k|}\left(\frac{\partial}{\partial y_{1}}\right)^{k_{1}} \cdots$ $\left(\frac{\partial}{\partial y_{n-1}}\right)^{k_{n-1}}$.

Definition 2.2. A distribution $u(x, y)$ on $R^{n}$ is said to be in $W^{m}\left(R^{n}, \tilde{\phi}\right)$ if and only if

$$
\|u\|_{m, \bar{\phi}}^{2}=\sum_{j,|k| \zeta m} \int_{R^{n}}\left|D_{\bar{\rho}}^{\frac{1}{\rho}} D_{v}^{k} u\right|^{2} d \bar{\phi} d y
$$

is finite, where $D_{\tilde{\rho}}$ stand for $-i \tilde{p}(x) \frac{\partial}{\partial x}$. 
On Dirichlet problems for some degenerate elliptic equations 379

Clearly $W^{m}\left(R_{+}^{n}, \phi\right)$ and $W^{m}\left(R^{n}, \tilde{\phi}\right)(m=0,1, \cdots)$ make Hilbert spaces by the norms (2.3) and (2.4) and we can regard $W^{m}\left(R^{n}, \tilde{\phi}\right) \subset W^{m}\left(R_{+}^{n}, \phi\right)$ by the natural restriction.

Let us define the weighted Fourier transformation $\mathscr{F}_{\bar{p}}$ and the tangential Fourier transformation $\mathscr{F}_{*}$ by

$$
\begin{aligned}
& \left(\mathscr{F}_{\tilde{p}} u\right)(\xi, \eta)=\int_{R^{n}} e^{-z z i \tilde{j} \tilde{x} ; \xi^{-z z i y \cdot \eta}} u(x, y) d \tilde{\phi} d y \\
& \left(\mathcal{F}_{,} u\right)(x, \eta)=\int_{R^{n-1}} e^{-z z i y \cdot \eta} u(x, y) d y
\end{aligned}
$$

where $y \cdot \eta=\sum_{j=1}^{n-1} y_{j} \eta_{j}$.

Remark. We could understand $W^{m}\left(R_{+}^{n}, \phi\right)$ (resp. $W^{m}\left(R^{n}, \tilde{\phi}\right)$ ) in the following way: consider the correspondence between $u(\dot{x}, y)$ and $v(x, y)$ defined by $\hat{x}=\phi(x)$ (resp. $\tilde{\phi}(x))$ and $(v(x, y)=u(\phi(x), y)$. $\hat{x}=\phi(x)$ defines a one-to-one correspondence between $R_{+, x}^{1}$ (resp. $R_{x}^{1}$ ) and $R_{+, x}^{1}$ (resp. $R_{x}^{1}$ ). Moreover we have

$$
\frac{\partial}{\partial \dot{x}} u(\hat{x}, y)=\rho(x) \frac{\partial}{\partial x} v(x, y)
$$

and we have

$$
\int_{R_{+}^{n}}|u(\dot{x}, y)|^{2} d \hat{x} d y=\int_{R_{+}^{n}}|v(x, y)|^{2} d \phi d y .
$$

Thus we can say that, in order that $u(x, y)$ belongs to $W^{m}\left(R_{+}^{n}, \phi\right)$ (resp. $W^{m}\left(R^{n}, \tilde{\phi}\right)$ ), it is necessary and sufficient that $u(\hat{x}, y) \in H^{m}\left(R_{+}^{n}\right)$ (resp. $H^{m}\left(R^{n}\right)$ ), the usual Sobolev space, where $v(x, y)=u(\phi(x), y)$ (resp. $u(\tilde{f}(x), y)$ ).

According to the above remark, the following two lemmas will be seen easily.

Lemma 2.1. $\mathscr{F}_{\tilde{\rho}}$ is a unitary transformation from $W^{0}\left(R^{n}, \bar{\phi}\right)$ onto $L^{2}\left(R^{n}\right)$ and the inverse transformation $\mathscr{F}_{\bar{p}}^{-1}$ is given by

$$
\left(\mathscr{F}_{\tilde{\rho}}^{-1} v\right)(x, y)=\int_{R^{n}} e^{2 z i \bar{\phi}(x) \xi+3 z i y \cdot \eta} v(\xi, \eta) d \xi d \eta .
$$

Lemma 2.2. Let $u(x, y)$ be in $W^{m}\left(R^{n} . \tilde{\phi}\right)$, then we have

$$
\left[\mathscr{T}_{\bar{p}}\left(D_{\bar{\rho}}^{\jmath} D_{s}^{\prime} u\right)\right](\xi, \eta)=(2 \pi \xi)^{j}(2 \pi \eta)^{k}\left(\mathcal{F}_{\bar{\rho}} u\right)(\xi, \eta) \text { if } j+|k| \leqq m .
$$


The following lemma guarantees that the element in $W^{m}\left(R_{+}^{n}, \phi\right)$ $(m \geqq 1)$ admits the trace to $x=0$.

Lemma 2. 3. If $u(x, y)$ belongs to $W^{\prime}\left(R_{+}^{\mathrm{n}}, \phi\right)$, then the trace $u(0, y)\left(=\lim _{x \neq 0} u(x, y)\right)$ exists in $H^{1 / 2}\left(R^{n-1}\right)$ and it holds

(2.9) $\quad|u(0, \cdot)|_{0}^{2} \leqq \varepsilon|| u\left\|_{1, \phi}^{2}+\varepsilon^{-1}|| u\right\|_{0, \phi}^{2} \quad$ for $\quad \forall \varepsilon>0$

(2.10) $\quad|u(0, \cdot)|_{\mathrm{i} / 2}^{2} \leq C|| u \|_{1, \phi}^{2}$

where $|\cdot|$ s is the norm in $H^{s}\left(R^{n-1}\right)$.

Proof. From the definition of $W^{1}\left(R_{+}^{n}, \phi\right)$, it follows

$$
u(x, y)-u\left(x^{\prime}, y\right)=\int_{x^{\prime}}^{x} \frac{\partial}{\partial} \xi \xi \xi\left(x, x^{\prime}>0\right) \text { for }(a . e) y,
$$

hence by Schwarz' inequality we have

$$
\left|u(x, y)-u\left(x^{\prime}, y\right)\right|^{2} \leqq\left|\phi(x)-\phi\left(x^{\prime}\right)\right| \int_{0}^{\infty}\left|D_{p} u\right|^{z} d \phi
$$

thus we have the trace in $L^{2}\left(R^{n-1}\right) .(2.8)$ and (2.9) are obtained by the following relation

(2.12) $|\hat{u}(0, \eta)|^{2}=2 \operatorname{Im} \int_{0}^{\infty} D_{\rho} \hat{u}(x, \eta) \cdot \overline{\hat{u}(x, \eta)} d \phi$

where $\hat{u}$ denotes the tangential Fourier image of $u$. (Q.E.D.)

Corollary 2.1. If $u(x, y) \in W^{m}\left(R_{+}^{n}, \phi\right)$, then all the traces of $D_{\beta}^{\prime} D_{y}^{k} u(j+|k| \leqq m-1)$ exist in $\left.H^{m-j-1}\right|^{-1 / 2}\left(R^{n-1}\right)$ and it holds

(2.13) $\left|D_{\rho}^{J} D_{v}^{k} u(0, \cdot)\right|_{s} \leqq c(j, k, m)\left(\varepsilon\left|\|u\|_{w_{, \phi}}^{2}+c(\varepsilon)\right|\left\{u \|_{0, \phi}^{2}\right)\right.$

$$
(s<m-j-|k|-1 / 2) \text { for }{ }^{*} \varepsilon>0
$$

(2.14) $\left|D_{\rho}^{J} D_{v}^{k} u(0, \cdot)\right|_{m-j-|k|-1 / 2} \leq C|| u \|_{m, \phi}^{2}$

In the case of the weighted Sobolev spaces, we obtain also the interpolation theorem similar to the case of the usual Sobolev spaces.

Proposition 2.1. (Interpolation theorem) 1) Let $u(x, y)$ and $D_{\rho}^{n} u(x, y)$ belong to $W^{\circ}\left(R_{+}^{n}, \phi\right)$, then $D_{\rho}^{k} u \in W^{\circ}\left(R_{+}^{n}, \phi\right)(1 \leqq k \leqq m-1)$ and we have

$$
\left\|D_{\rho} u_{\rho}^{k}\right\|_{0, \uparrow} \leqq C(k, m)\left(\varepsilon\left\|D_{\rho}^{m} u\right\|_{0, \downarrow}+C(\varepsilon) \mid u \|_{0, \downarrow}\right) \text { for } \quad{ }^{v} \varepsilon>0 .
$$


2) If $D_{y}^{k} u(|k| \leqq m)$ and $D_{\beta}^{m} u$ belong to $W^{\circ}\left(R_{+}^{n}, \phi\right)$, then $u \in W^{m}\left(R_{+}^{n}, \phi\right)$ and we obtain for ${ }^{\vee} E>0$

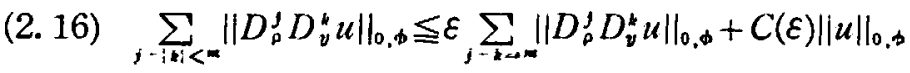

Proof. 1) It will be sufficient to prove in the case when $m=2$ and $k=1$. First we note

$$
D_{\rho} u(x, y)=\left(D_{\rho} u\right)(0, y)+\int_{0}^{x} D_{\rho}^{2} u(\xi, y) d \phi(\xi),
$$

thus we can see $D_{\rho} u$ is square integrable in $d \phi d y$ on $(0,1) \times R^{n-1}$. Now $\beta(x)$ be an infinitely differentiable function on $R^{1}$ which vanishes identically for $x \leqq 0$ and identically equals to one for $x \geqq 1$, then $D_{\tilde{\tilde{p}}}^{\tilde{z}}(\beta u)$ and $\beta u$ belong to $W^{\mathrm{o}}\left(R^{n}, \tilde{\phi}\right)$ and consequently $\left(1+\xi^{2}+|\eta|^{2}\right) \mathscr{F}_{\bar{p}}(\beta u)$ does to $L^{2}\left(R^{n}\right)$. Hence we can see that $D_{\bar{\phi}}(\beta u)$ is in $W^{n}\left(R^{n}, \phi\right)$ and therefore $D_{\rho} u$ in $W^{\circ}\left(R_{+}^{n}, \phi\right)$. (2.15) is shown as follows: at first by the integration by parts, we have

$$
\int_{R_{+}^{n}}\left|D_{\rho} u\right|^{2} d \phi d y=\int_{R_{+}^{n}} u \cdot \overline{D_{\rho}^{n} u} d \phi d y-\int_{R^{n-1}} u(0, y) \overline{D_{\rho}} u(\overline{0, y}) d y,
$$

then by Lemma 2.3 we can obtain (2.15) easily.

2) It will suffice to show in the case when $m=2$ and $k=1$ also.

Note at first

$$
\dot{u}(x, \eta)=\dot{u}(0, \eta)+i \int_{0}^{x} D_{\mathrm{p}} \dot{u} d \phi
$$

hence multiplying $|\eta|$ on both sides and using the Schwartz' inequality we obtain

$$
\begin{aligned}
& \int_{0}^{\delta} \int_{R^{n-1}}|\eta|^{2}|\hat{u}(x, \eta)|^{2} d \eta d \phi \leqq 2 \phi(\delta)|u(0, \cdot)|_{1}^{2} \\
& \quad+\phi(\delta)^{2} \int_{R_{+}^{n}}\left|I_{\rho} \dot{u}\right|^{2}|\eta|^{2} d \phi d \eta .
\end{aligned}
$$

On the other hand for fixed $\delta>0$, it is well-known that

$$
\begin{gathered}
\int_{\delta}^{\infty} \int_{R^{n-1}}|\eta|^{2}|\hat{u}|^{2} d \eta d \phi \leqq \varepsilon \int_{R_{+}^{n}}\left|D_{\rho} \dot{u}\right|^{2}|\eta|^{2} d \phi d \eta+C(\varepsilon) \\
\int_{R_{+}^{n}}|\dot{u}|^{2} d \phi d \eta
\end{gathered}
$$


is valid. Thus taking $\delta>0$ suitably and applying Lemma 2.3., we can get (2.16).

(Q.E. D.)

We state here a proposition which will be needed to deal with first order terms.

Lemma 2. 4. If $u \in W^{2}\left(R_{+}^{\mathrm{n}}, \phi\right)$ with $\alpha<\frac{1}{3}$, then $\frac{\partial u}{\partial x}$ belong to $W^{\circ}\left(R_{+}^{n}, \phi\right)$ and moreover it holds for ${ }^{\vee} \varepsilon>0$

$$
\left\|\frac{\partial u}{\partial x}\right\|_{0, \phi} \leqq \varepsilon\|u\|_{2, \phi}+C(\varepsilon)\|u\|_{0, \phi}
$$

Proof. Since $\rho(x)$ vanishes only at $x=0$, by the well-known estimate in the case of the usual Sobolev space we have for any $u \in W^{2}\left(R_{+}^{n}, \phi\right)$

$$
\int_{R}^{\infty} \int_{R^{n-1}}\left|\frac{\partial u}{\partial x}\right|^{2} d \phi d y \leqq \delta \int_{R_{+}^{n}}\left|D_{\rho}^{2} u\right|^{2} d \phi d y+C(\delta) \int_{R_{+}^{n}}|u|^{2} d \phi d y
$$

where $R$ is a positive number and $\delta$ is an arbitrary positive number.

Now for ${ }^{v} u \in W^{2}\left(R_{+}^{n}, \phi\right)$ we have

$$
\left|D_{\rho} u(x, y)\right|^{2}=\left|D_{\rho} u(0, y)\right|^{2}+2 R e \int_{0}^{x} D_{\rho}^{2} u \cdot \overline{D_{\rho} u} d \phi
$$

hence

$$
\begin{aligned}
& \left|\frac{\partial u}{\partial x}\right|^{2} \leqq \frac{1}{\rho^{2}(x)}\left|D_{\rho} u(0, y)\right|^{2}+\frac{1}{\rho^{2}(x)}\left(\varepsilon_{1} \int_{0}^{\infty}\left|D_{\mathrm{\rho}}^{2} u\right|^{2} d \phi\right. \\
& \left.\quad+\frac{1}{\varepsilon_{1}} \int_{0}^{\infty}\left|D_{\rho} u\right|^{2} d \phi\right),
\end{aligned}
$$

thus if $\alpha<\frac{1}{3}$ we obtain

$$
\begin{aligned}
& \int_{0}^{R} \int_{R^{n-1} \mid}\left|\frac{\partial u}{\partial x}\right|^{2} d \phi d y \leqq \int_{0}^{R} \frac{d x}{\rho^{3}(x)}\left(\int_{R^{n-1}}\left|D_{\rho} u(0, y)\right|^{2} d y\right. \\
& \left.\quad+\varepsilon_{1} \int_{R_{+}^{n}}\left|D_{\ominus}^{2} u\right|^{2} d \phi+\varepsilon_{1}^{-1} \int_{0}^{\infty}\left|D_{\rho} u\right|^{2} d \phi\right)
\end{aligned}
$$

Together with (2.21), (2.24) Corollary 2.1 and Proposition 2.1, we obtain (2.20). (Q. E. D.) 
On Dirichlet problems for some degenerate elliptic equations 383

Remark. If $\alpha \geqq 1 / 3$, we can find a function in $W^{2}\left(R_{+}^{n}, \phi\right)$ such that $\partial u / \partial x$ does not belong to $W^{\circ}\left(R_{+}^{n}, \phi\right)$. For example, take the function which is equal to $\phi(x) g(y)$ near $x=0$, where $g(y) \in L^{2}\left(R^{n-1}\right)$.

Let us remark here that $D_{\rho}$ is formally self-adjoint with respect to the inner product of $W^{\circ}\left(R_{+}^{n}, \phi\right)$, whereas the formal adjoint of $\frac{1}{i} \frac{\partial}{\partial x}$ in $W^{\circ}\left(R_{+}^{n}, \phi\right)$ is given by

$$
\left(\frac{1}{i} \frac{\partial}{\partial x}\right)^{*}=\frac{1}{i} \cdot \frac{\partial}{\partial x}+\frac{1}{i}-\frac{d \rho}{d x} \cdot \frac{1}{\rho}
$$

and the second term of the right hand of (2.25) will behave like $x^{-1}$ near $x=0$.

Definition 2.3. We denote by $W_{0}^{m}\left(R_{+}^{n}, \phi\right)$ the completion of $C_{0}^{\infty}\left(R_{+}^{n}\right)$ in $W^{m}\left(R_{+}^{n}, \phi\right)$.

Using Corollary 2.1, we have immediately

Proposition 2.2. Suppose $u(x, y) \in W_{0}^{m}\left(R_{+}^{n}, \phi\right)$. The traces of . $D_{\rho}^{\prime} D_{v}^{*} u(j+|k| \leqq m-1)$ are all equal to zero.

Remark. We have the following algebraic and topological inclusion relations between our weighted Sobolev spaces and the usual ones :

$$
\left\{\begin{array}{l}
W^{m}\left(R_{+}^{n}, \phi\right) \subset H^{m}\left(R_{+}^{n}\right) \\
C_{0}^{m}\left(R_{+}^{n}\right) \subset W_{0}^{m}\left(R_{+}^{n}, \phi\right) \subset \mathcal{D}_{L^{2}}^{m_{2}}\left(R_{+}^{n}\right)
\end{array}\right.
$$

To deal with $\frac{d \rho}{d x} \cdot \frac{1}{\rho}$, we prepare the following

Lemma 2. 5. Let $u$ be in $W^{2}\left(R_{+}^{n}, \phi\right) \cap W_{0}^{1}\left(R_{+}^{n}, \phi\right)$, then $x^{-1} u$ belongs to $W^{\circ}\left(R_{+}^{n}, \phi\right)$ if $\alpha<1 / 3$, and it holds

(2.27) $\quad \mid i x^{-1} u\left\|_{0, \phi} \leqq £\right\| u\left\|_{2, \phi}+C(\varepsilon)\right\| u \|_{0, \phi}$ for ${ }^{\vee} \varepsilon>0$. Moreover, if $\geq \alpha 1 / 3$ we can find $u$ in $W^{2}\left(R_{+}^{n}, \phi\right)$ $\cap W_{0}^{1}\left(R_{+}^{n}, \phi\right)$ such that $x^{-1} u$ does not belong to $W^{\circ}\left(R_{+}^{n}, \phi\right)$.

Proof. By considering the remark of page 379 , we have the following Taylor expansion of $u(x, y)$; 
(2.28) $\quad u(x, y)=\left(\rho \frac{\partial u}{\partial x}\right)(0, y) \phi(x)+\int_{0}^{x}\left(\left(\phi(x)-\phi\left(x^{\prime}\right)\right)\right.$

$$
\left(\rho \frac{\partial}{\partial x^{\prime}}\right)^{2} u\left(x^{\prime}, y\right) d \phi\left(x^{\prime}\right) \text {. }
$$

By the assumptions on $\rho(x)$ we see near $x=0$

$$
\begin{aligned}
& \left|\frac{u(x, y)}{x}\right|^{2} \frac{1}{\rho(x)} \leqq C\left(x^{-3 \alpha}\left|\left(\rho \frac{\partial u}{\partial x}\right)(0, y)\right|^{2}\right. \\
& \left.+x^{1-\alpha \alpha} \int_{0}^{\infty}\left|D_{\rho}^{2} u\right|^{2} d \phi\right)
\end{aligned}
$$

hence we have for an arbitrary $\delta>0$

$$
\begin{aligned}
& \int_{0}^{\delta} \int_{R^{n-1}}\left|\frac{u}{x}\right|^{2} d \phi d y \leqq C(\alpha)\left(\delta^{1-3 \alpha}\left|D_{\rho} u(0, y)\right|_{0}^{2}\right. \\
& \left.\quad+\delta^{2--\alpha} \int_{0}^{\infty}\left|D_{\rho}^{2} u\right|^{2} d \phi\right) .
\end{aligned}
$$

On the other hand it holds

$$
\int_{8}^{\infty} \int_{R^{n-1}}\left|\frac{u}{x}\right|^{2} d \phi d y \leqq \frac{1}{\delta^{2}} \int_{R_{+}^{n}}|u|^{2} d \phi d y
$$

Thus togethdr with (2.30) and (2.21), and using Corollary 2.1., we can show (2.21). For the last part, see the remark at page 383 .

(Q. E. D.)

Lemma 2. 6. If $u(x, y) \in W^{2}\left(R_{+}^{n}, \phi\right) \cap W_{0}^{1}\left(R_{+}^{n}, \phi\right)$, then $x^{-\beta} \frac{\partial u}{\partial y_{j}}$ $(j=1, \cdots, n-1)$ belong to $W^{\circ}\left(R_{+}^{n}, \phi\right)$ when $\beta<1-\alpha$, and it holds for ${ }^{\vee} \varepsilon>0$

$$
\left\|x^{-\beta} \cdot \frac{\phi u}{\phi y_{j}}\right\|_{0, \phi} \leqq \varepsilon\|u\|_{0, p}+C(\xi)\|u\|_{0, \phi}
$$

Proof. By the Taylor expansion we obtain

$$
\left.\frac{\partial u}{\partial y_{j}}(x, y)=\int_{0}^{x}\left(\rho \frac{\partial}{\partial x}\right)^{\prime} \frac{\partial u}{\partial y_{j}}\right) d \phi
$$

hence near $x=0$

$$
x^{-2 \beta}\left|\frac{\partial u}{\partial y_{j}}\right|^{2} \frac{1}{\rho(x)} \leqq C x^{1-2 \beta-2 \alpha} \int_{0}^{\infty}\left|D_{\rho} \frac{\partial u}{\partial y_{j}}\right|^{2} d \phi
$$


and since $\beta<1-\alpha$, we can show (2.32) by the similar manner to that of the proof of Lemma 2.5.

(Q. E. D.)

At the end of this section we introduce some families of functions.

Definition 2.4. We say that a function $a(x, y)$ on $R_{+}^{n}$ belong to $\mathcal{B}^{m}\left(R_{+}^{n}, \rho\right)$ if and only if $D_{\rho}^{s} D_{v}^{k} a(j+|k| \leqq m)$ are continuous and bounded.

It should be mentioned that $\mathcal{B}^{m}\left(R_{1}^{n}, \rho\right)$ has sufficiently many elements. In fact, if $\alpha(x, y) \in \mathscr{B}^{m}\left(R_{+}^{n},\right)$ then $a(x, y)=\alpha(\phi(x), y)$ belong to $\mathcal{B}^{m}\left(R_{+}^{n}, \rho\right)$ and $\varphi(x, y)+$ const. $\left(\varphi \in C_{0}^{\infty}\left(R_{+}^{n}\right)\right)$ also belong to $\mathcal{B}^{m}\left(R_{+}^{\mathrm{n}}, \rho\right)$.

3. Sobolev spaces with weights in general domain $\Omega$. Let $\Omega \subset R^{n}$ be a domain whose boundary is a smooth compact hyper surface $\Gamma$. In this section we introduce the Sobolev spaces with weights in $\Omega$, which are closely related to $W^{m}\left(R_{\downarrow}^{n}, \phi\right)$, in which we treat our problems, and study their basic properties.

We start with the following

Definition 3. 1. Let $\omega$ be a sufficiently small closed neighbourhood of some boundary point where we are allowed to consider $\frac{\partial}{\partial \nu}$ and $\frac{\partial}{\partial \tau}$. A function $a(x)$ on $\omega$ is said to be in $\mathscr{B}^{m}(\omega, \rho)$ if and only if $\left(\rho(r) \frac{\partial}{\partial \nu}\right)^{j}\left(-\frac{\partial}{\partial \tau}\right)^{k} a(x)(j+|k| \leqq m)$ and continuous and bounded.

Remark. $a(x) \in \mathscr{B}^{m}(\omega, \rho)$ can be constructed, for example, as follows: let $\alpha(\nu, \tau)$ be in $\mathscr{B}^{m}\left(R_{+}^{n}, \rho\right)$ with small support, then $a(x)=\alpha \circ \theta^{-1}(\nu, \tau) \in \mathscr{B}^{m}(\omega, \rho)$, where $\theta^{-1}$ is the inverse transformation of $\theta: x \rightarrow(\nu, \tau)$ defined by (1.4).

Now take a finite covering $\left\{\omega_{p}\right\}$ of $\Omega$ such that ;

1) if we denote $\omega_{\lambda}^{*}$ the covering neighbourhood which contact with $\Gamma$, then $\cup \omega_{\lambda}^{*} \supset \bar{\Omega}_{\varepsilon}$, with small $\varepsilon>0$,

2) for the all inner coverings, we denote them by $\left\{\tilde{\omega}_{\mu}\right\}$, all the supports of them lie in $\Omega_{\mathfrak{g} / 2}^{\mathrm{C}}$ where $\Omega_{\gamma}=\{x \in \bar{\Omega} ; \operatorname{dis}(x, \Gamma)<\gamma\}$, and then take a partion of unity 
$\left\{\boldsymbol{\rho}_{\Lambda}^{*}\right\} \cup\left\{\tilde{\Phi}_{\mu}\right\}$ on $\bar{\Omega}$ such that :

1) supp. $\varphi_{\lambda}^{*} \subset \omega_{\lambda}^{*}\left(\omega_{\lambda}^{*}\right.$ 's are sufficiently small where we can consider $\frac{\partial}{\partial \nu}$ and $\left.\frac{\partial}{\partial \tau}\right)$ and supp. $\tilde{\mathcal{P}}_{\mu} \subset \tilde{\omega}_{\mu}$

2) $\sum \varphi_{\lambda}^{* 2}+\sum \tilde{p}_{\mu}^{2}=1$, and $\varphi_{\lambda}^{*} \in \mathscr{B}^{\infty}\left(\omega^{*}, \rho\right)$ and $\tilde{p}_{\mu} \in C_{0}^{\infty}\left(\tilde{\omega}_{\mu}\right)$.

Let us define our weighted Soboldv spaces.

Definition 3.2. A distribution $u(x)$ on $\Omega$ is said to be in $W^{m}(\Omega, \rho)(m$ : non-negative integer) if and only if

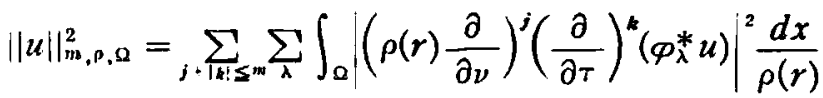

$$
\begin{aligned}
& +\sum_{1 \mid i \leq m} \sum_{\mu} \int_{0} \mid\left(\frac{\partial}{\partial x}\right)^{l}\left(\widetilde{\mathcal{P}}_{\mu} u\right)^{i 2} \frac{d x}{\rho(r)}
\end{aligned}
$$

is finite.

The norm given by (3.1) depends on $\varepsilon$ and the choice of partition of unity, but we shall continue our arguments with fixed $\varepsilon$ sufficiently small for which we can consider $\partial / \partial p$ and $\partial / \partial \tau$ in $\Omega_{z z}$ and since the passage to another choice of $\left\{\omega_{\lambda}^{*}\right\}$ and $\left\{\tilde{\omega}_{\mu}\right\}$, consequently $\left\{\varphi_{\lambda}^{*}\right\}$ and $\left\{\tilde{\boldsymbol{P}}_{\mu}\right\}$, gives an equivalent norm, we shall always mean by $\|u\|_{m, \rho, \Omega}$ an arbitrary but fixed norm defining the $W^{m}(\Omega, \rho)$ topology in what follows.

We denote by $W_{0}^{m}(\Omega, \rho)$ the completion of $C_{0}^{\infty}(\Omega)$ in $W^{m}(\Omega, \rho)$. Clearly $W^{m}(\Omega, \rho)$ and $W_{0}^{m}(\Omega, \rho)(m=0,1, \cdots)$ make Hilbert spaces by the norm $\|u\|_{m, p, \alpha}$.

Let us remark here that by the transformation (1.4), each small boundary patch is mapped into $\overline{R_{+}^{n}}$ and $\partial / \partial \nu$ and $\partial / \partial \tau$ are transformed to the normal and the tangential differentiations in $R_{+}^{n}$ respectively, thus $W^{m}(\omega, \rho)$ is transformed into $W^{m}\left(R_{+}^{n}, \phi\right)$ with equivalent norms, where $\omega$ denotes a sufficiently small boundary patch.

Hence taking account of Corollary 2.1, Lemma 2.4, Lemma 2.5. and Lemma 2.6., we have

Proposition 3. 1. Any $u(x) \in W^{m}(\Omega, \rho)$ admits the trace $\gamma D^{\beta} u$ in $H^{m-191-1 / 2}(\Gamma)$ and it holds

$$
\left|\gamma D^{\beta} u\right|_{s, \Gamma \leqq} \leqq\left.\left.\right|_{\mid u}\right|_{m, \rho, Q}+\left.C(\varepsilon)|| u\right|_{\text {o,p,Q }} \quad(s<m-\mid \beta-1 / 2)
$$


On Dirichlet problems for some degenerate elliptic equations 387

$$
\left|\gamma D^{\beta} u\right|_{m-\left.1 \beta|-1 / 2, \Gamma \leqq C i| u\right|_{m, \rho, Q}}
$$

for ${ }^{{ }} \varepsilon>0$, where $D^{\beta}=\left(\rho(r) \frac{\partial}{\partial \nu}\right)^{\beta_{1}}\left(\frac{\partial}{\partial \tau}\right)^{\beta_{3}} \quad\left(|\beta|=\beta_{1}+\left|\beta_{2}\right|<m\right)$ and $|\cdot|_{s, \Gamma}$ denotes the norm in $H^{s}(\Gamma)$.

Lemma 3. 1. Let $u(x)$ be in $W^{2}(\Omega, \rho)$ and $\alpha<\frac{1}{3}$, then $\frac{\partial u}{\partial x_{j}}$ $(j=1, \cdots, n)$ are all in $W^{\circ}(\Omega, \rho)$, and it holds for ${ }^{v} \varepsilon>0$

$$
\left\|\frac{\partial u}{\partial x_{j}}\right\|_{0, \rho, \Omega} \leqq \varepsilon\|u\|_{2, \rho, Q}+C(\varepsilon)\|u\|_{0, \rho, \Omega}
$$

Lemma 2.2. If $u(x) \in W^{2}(\Omega, \rho) \cap W_{0}^{1}(\Omega, \rho)$ with $\alpha<\frac{1}{3}$ then $u / r \in W^{\circ}(\Omega, \rho)$ and we have with ${ }^{\vee} \varepsilon>0$

$$
\|u / r\|_{0, P, Q} \leqq \varepsilon\|u\|_{2, p, Q}+C(\varepsilon)\|u\|_{0, p, Q} \text {. }
$$

and finally

Lemma 3. 3. Let $u(x)$ be in $W^{2}(\Omega, \rho) \cap W_{0}^{1}(\Omega, \rho)$, then for each $\omega_{\lambda}^{*}$ we have with $\beta<1-\alpha$

$$
\int_{\omega_{\lambda}^{*} \cap \Omega}\left|r^{-\beta} \frac{\partial u}{\partial \tau}\right|^{2} \frac{d x}{\rho(r)} \leqq \varepsilon\|u\|_{2, \rho, \Omega}^{2}+C(\varepsilon)\|u\|_{0, \rho, \alpha}^{2} .
$$

The proof of above proposition and lemma will be carried out by the localization by the partition of unity of class $\mathcal{B}^{\infty}(\omega, \rho)$ near the boundary and by the local transformation (1.4).

4. Dirichlet problems for second order equations. In this section we shall treat the Dirichlet problems with homogeneous boundary condition:

$$
\left\{\begin{aligned}
A u & =-\sum_{j, k+1}^{n} a_{j k}(x) \frac{\partial^{2} u}{\partial x_{j}} \frac{\partial x_{k}}{\partial x_{j=1}^{n}} b_{j}(x) \frac{\partial u}{\partial x_{j}}+c(x) u \\
& =f(x) \in W^{0}(\Omega, \rho) \\
\left.u\right|_{\Gamma} & =0
\end{aligned}\right.
$$

where we assume (1.3) for $A$, more presicely $A$ can be written in each $\omega_{\lambda}^{*}$ in the following form 
(4. 2)

$$
\begin{aligned}
A u & =-\sum_{j, k=0}^{n-1} \tilde{a}_{j k}(x) \partial_{j} \partial_{k}+\sum_{j=0}^{n-1} \tilde{b}_{j} \partial_{j}+\tilde{c}_{0}(x) \frac{\partial}{\partial \nu} \\
& +\sum_{j=1}^{n-1} \tilde{c}_{j}(x) r^{-\beta} \partial_{j}+\tilde{d}_{0}(x) \frac{1}{r}+d(x)
\end{aligned}
$$

with $\sum \bar{a}_{j k} \xi_{j} \xi_{k} \geqq c \sum \xi_{j}^{2}\left(\check{a}_{j k}=\overline{\hat{a}_{k j}}\right)$ and $\beta>1-\alpha$, where $\hat{a}_{j k} \in \mathscr{B}^{j}\left(\omega_{\lambda}^{*}, \rho\right)$ $b_{j}(x)(j=0,1, \cdots, n-1)$ and $\tau_{j}(x)(j=0, \cdots, n-1) \in \mathscr{B}^{1}\left(\omega_{\lambda}^{*}, \rho\right)$ and $\tilde{d}_{0}(x), \tilde{d}(x) \in \mathcal{B}^{0}\left(\omega_{\lambda}^{*}, \rho\right)$.

The following lemma is needed to show the Gàrding inequality.

Lemma 4. 1. Let $u(x)$ be in $W_{0}^{1}(\Omega, \rho)$ with $\alpha<\frac{1}{2}$, then we have

$$
\int_{\Omega}\left|\frac{\partial u}{\partial x_{j}} u\right| \frac{d x}{\rho(r)} \leqq \varepsilon\|u\|_{1, \rho, \Omega}^{2}+C(\varepsilon)\|u\|_{0, \rho, \Omega}^{2} \quad(j=1, \cdots, n)
$$

$$
\int_{0} \frac{|u|^{2}}{r} \frac{d x}{\rho(r)} \leqq \varepsilon\|u\|_{1, \rho, Q}^{2}+C(\varepsilon)\|u\|_{0, \rho, Q}^{2}
$$

and for each $\omega_{\lambda}^{*}$

$$
\int_{\operatorname{Ln} \cap \omega_{\lambda}^{*}} r^{-\beta}\left|\frac{\partial u}{\partial \tau} u\right| \frac{d x}{\rho(r)} \leqq \varepsilon\|u\|_{1, \rho, \Omega}^{2}+C(\varepsilon)\|u\|_{0, \rho, \Omega}^{2} \quad(\beta<1-\alpha)
$$

for ${ }^{v_{E}}>0$.

The above lemma is the consequence of the following lemma,

Lemma 4.2. Let $u(x, y)$ be in $W_{0}^{2}\left(R_{+}^{n}, \phi\right)$ with $\alpha<1 / 2$, then we obtain

$$
\int_{R_{+}^{n}}\left|\frac{\partial u}{\partial x} u\right| d \phi d y \leqq \varepsilon\|u\|_{i, \phi}^{2}+C(\varepsilon)\|u\|_{0, \phi}^{2}
$$

$$
\int_{R_{+}^{n}} \frac{|u|^{2}}{x} d \phi d y \leqq \varepsilon\|u\|_{1, \phi}^{2}+C(\varepsilon)\|u\|_{0, \phi}^{2}
$$

and

$$
\int_{R_{+}^{n}} x^{-\beta}\left|\frac{\partial u}{\partial y_{j}} u\right| d \phi d y \leq \varepsilon\|u\|_{1, \phi}^{2}+C(\varepsilon)\|u\|_{0, \phi}^{2} \quad(\beta<1-\alpha)
$$

for ${ }^{\vee} \varepsilon>0$.

Proof. First we remark that it holds for $u \in W_{0}^{1}\left(R_{+}^{n}, \phi\right)$ $u(x, y)=\int_{0}^{x} \rho \frac{\partial u}{\partial x} d \phi \quad$ (a. e.) y. 
On Dirichlet problems for some degenerate elliptic equations 389 hence

$$
\text { (4. 9) }\left.\quad\left|u(x, y) !^{2} \leqq \phi(x) \int_{0}^{\infty}\right| D_{\rho} u\right|^{2} d \phi
$$

thus we havd near $x=0$

$$
\text { (4.10) } \frac{|u|^{2}}{\rho(x)^{3}} \leqq \phi(x) \rho(x)^{-3} \int_{0}^{\infty}\left|D_{\rho} u\right|^{2} d \phi \leqq C x^{1-\alpha u} \int_{0}^{\infty}\left|D_{\mathrm{p}} u\right|^{2} d \phi \text {. }
$$

Since $\alpha<1 / 2$, from (4.10) we obtain for any small $\delta>0$

(4. 11) $\int_{0}^{\delta} \frac{|u|^{2}}{\rho^{2}(x)} d \phi \leqq C(\alpha) \delta^{2-\iota x} \int_{0}^{\infty}\left|D_{\rho} u\right|^{2} d \phi$

thus we have

$$
\begin{aligned}
& \int_{R_{+}^{n}} \frac{\mid u_{\rho^{2}}^{2}(x)}{2} d \phi d y \leq C(\alpha) \delta^{2-\alpha \alpha} \int_{R_{+}^{n}}\left|D_{\rho} u\right|^{2} d \phi d y \\
& \quad+K(\delta, \alpha) \int_{R_{+}^{n}}|u|^{2} d \phi d y .
\end{aligned}
$$

On the other hand it holds for ${ }^{\mathrm{v}} \varepsilon_{1}>0$

$$
\int_{R_{+}^{n}}\left|\frac{\partial u}{\partial x} u\right| d \phi d y \leq \varepsilon_{1} \int_{R_{+}^{n}}\left|D_{\rho} u\right|^{:} d \phi d y+\frac{1}{\varepsilon_{1}} \int_{R_{+}^{n}} \frac{|u|^{2}}{\rho^{2}(x)} d \phi d y .
$$

Since we can take $\delta$ and $\varepsilon_{1}$ arbitrarily small, we obtain (4.6). Next from (4.9) we get near $x=0$

(4. 13) $\frac{|u|^{2}}{x \rho(x)} \leqq \frac{\varphi(x)}{x \rho(x)} \int_{0}^{\infty}\left|D_{\rho} u\right|^{2} d \phi \leqq C x^{-2 x} \int_{0}^{\infty}\left|D_{\rho} u\right|^{2} d \phi$

hence

$$
\text { (4. 14) } \int_{0}^{\delta} \frac{|u|^{2}}{x} d \phi \leqq C \delta^{1-2 s} \int_{0}^{\infty}\left|D_{\rho} u\right|^{2} d \phi
$$

thus we obtain

$$
\text { (4.15) } \int_{R_{+}^{n}} \frac{|u|^{2}}{x} d \phi d y \leqq C \delta^{1-z \alpha} \int_{R_{+}^{n}}\left|D_{\mathrm{\rho}} u\right|^{2} d \phi+\frac{1}{\delta} \int_{R_{+}^{n}}|u|^{2} d \phi d y
$$

and this shows that (4.7) is valid. Finally we show (4.8). By Schwarz' inequality we have for an arbitrarily small $\delta>0$

$$
\int_{0}^{s}\left|x^{-\beta} \frac{\partial u}{\partial y_{j}} u\right| d \phi \leqq \sqrt{\left.\int_{0}^{\delta} \frac{\partial u}{\partial y_{j}}\right|^{2}} d \phi \sqrt{\int_{0}^{\delta} x^{-2 \beta}|u|^{2} d \phi},
$$

and taking account of (4.9) we obtain 


$$
x^{-2 \beta}|u|^{2} \leqq x^{-2 \beta} \phi(x) \int_{0}^{\infty}\left|D_{\rho} u\right|^{2} d \phi \leqq C x^{-\alpha-2 \beta} \int_{0}^{\infty}\left|D_{\rho} u\right|^{2} d \phi
$$

thus by (4.16) we get

$$
\int_{0}^{8}\left|x^{-\beta} \frac{\partial u}{\partial y_{j}} u\right| d \phi \leqq C \sqrt{\int_{0}^{\infty}\left|\frac{\partial u}{\partial y_{j}}\right|^{2} d \phi} \sqrt{\int_{0}^{\infty}\left|D_{\rho} u\right|^{2} d \phi} \sqrt{\int_{0}^{\delta} x^{1-2 \infty-2 \beta} d x}
$$

hence using Schwarz' inequality again we obtain

$$
\begin{aligned}
& \int_{0}^{\delta} \int_{R^{n-1}} x^{-\beta}\left|\frac{\partial u}{\partial y_{j}} u\right| d \phi \leqq C(\alpha, \beta) \delta^{2-2 \alpha-2 \beta}\left\|\frac{\partial u}{\partial y_{j}}\right\|_{0, \phi}\left\|D_{\rho} u\right\|_{0, \phi} \\
& \quad \leqq C(\alpha, \beta) \delta^{2-2 \alpha-2 \beta}\|u\|_{1, \phi}^{2}
\end{aligned}
$$

On the other hand it holds

$$
\begin{aligned}
& \int_{\delta}^{\infty} \int_{R^{n-1}} x^{-\beta}\left|\frac{\partial u}{\partial y_{j}} u\right| d \phi d y \leqq K(\delta) \int_{R_{+}^{n}}\left|\frac{\partial u}{\partial y_{j}} u\right| d \phi d y \\
& \quad \leqq K(\delta)\left(\varepsilon_{1}\|u\|_{1, \phi}^{2}+\frac{1}{\varepsilon_{1}}\|u\|_{0, \phi}^{2}\right)
\end{aligned}
$$

for ${ }^{n} \varepsilon_{1}>0$.

Thus taking $\delta$ and $\varepsilon_{1}$ suitably, we can get (4.8). (Q. E. D.)

In treating the Dirichlet problems our main tool will be the Gårding in equality which we state

Proposition 4.1. Assume that the coefficients of the principal part of $A$ are all in $C^{1}(\Omega)$ and others in $C^{0}(\Omega)$, and moreover near the boundary $\tilde{a}_{j k}(x) \in \mathscr{B}^{\prime}\left(\omega_{\lambda}^{*}, \rho\right)$, and $\tilde{b}_{j}(x)(j=0, \cdots, n-1), \tilde{c}_{j}(x)$ $(j=0, \cdots, n-1) d_{0}(x)$ and $\tilde{d}(x)$ are all in $\mathcal{B}^{\circ}\left(\omega_{\lambda}^{*}, \rho\right)$ for each $\omega_{\lambda}^{*}$, then there exist positive constants $C$ and $K$ such that

$$
\operatorname{Re}\langle A u, \bar{u}\rangle_{\rho, \Omega} \geqq C\|u\|_{1, \rho, Q}^{2}-K\|u\|_{0, \rho, \Omega}^{2}
$$

for all $u(x) \in W_{0}^{1}(\Omega, \rho)$ with $\alpha<1 / 2$, where $\langle,\rangle_{\rho, \Omega}$ denotes the sesquilinear form on $W_{0}^{1}(\Omega, \rho)^{\prime} \times W_{0}^{1}(\Omega, \rho)$ induced by the inner product of $W^{\circ}(\Omega, \rho)$. And the condition on $\alpha$ can not be weakened and $W_{0}^{1}(\Omega, \rho)^{\prime}$ denotes the dual space (which is a distributions space) of $W_{0}^{1}(\Omega, \rho)$.

Proof. Since $C_{0}^{\infty}(\Omega)$ is dense in $W_{0}^{1}(\Omega, \rho)$, it will suffices to show (4.28) for $u(x) \in C_{0}^{\infty}(\Omega)$. First we note 
On Dirichlet problems for some degenerate elliptic equations 391

$$
\langle A u, \bar{u}\rangle_{\rho, \Omega}=\sum_{\lambda} \int \varphi_{\lambda}^{*} A u \cdot \varphi_{\lambda}^{*} \bar{u} \frac{d x}{\rho(r)}+\sum_{\mu} \int \tilde{\mathcal{P}}_{\mu} A u \cdot \tilde{\mathcal{P}}_{\mu} \bar{u} \frac{d x}{\rho(r)} .
$$

Consider $\sum_{\mu} \int \tilde{\mathscr{P}}_{\mu} A u \cdot \widetilde{\mathcal{P}}_{\mu} \bar{u} \frac{d x}{\rho(r)}$. We write $\tilde{\mathscr{P}}_{\mu} A u=A\left(\tilde{\mathscr{P}}_{\mu} u\right)+\left[\tilde{\mathscr{P}}_{\mu}, A\right] u$. Since $\left[\mathscr{\psi}_{\mu}, A\right]$ is of first order operator and $\left[\widetilde{\mathcal{P}}_{\mu},\left[\mathscr{P}_{\mu}, A\right]\right]$ is of order zero, and since

$\int\left[\tilde{\mathcal{P}}_{\mu}, A\right] u \cdot \tilde{\mathcal{P}}_{\mu} \vec{u} \frac{d x}{\rho(r)}=\int\left[\tilde{\mathcal{P}}_{\mu}, A\right]\left(\tilde{\mathcal{P}}_{\mu} u\right) \tilde{u} \frac{d x}{\rho(r)}+\int\left[\tilde{\mathcal{P}}_{\mu},\left[\tilde{\mathcal{P}}_{\mu}, A\right]\right] \frac{|u|^{2}}{\rho(r)} d x$, we have

$$
\begin{aligned}
& \left|\sum_{\mu} \int\left[\widetilde{\mathscr{P}}_{\mu}, A\right] u \cdot \tilde{\mathcal{P}}_{\mu} \bar{u}-\frac{d x}{\rho}\right| \leqq \eta \sum_{i, \mu} \int\left|\frac{\partial}{\partial x_{j}}\left(\tilde{\mathcal{P}}_{\mu} u\right)\right|^{2} \frac{d x}{\rho} \\
& \quad+C(\eta) \int \frac{|u|^{2}}{\rho} d x
\end{aligned}
$$

where $\eta$ is an arbitrary positive number. Thus applying the result of regular case to $\sum_{\mu} \int A\left(\mathscr{\phi}_{\mu} u\right) \cdot \varphi_{\mu} \pi \frac{d x}{\rho(r)}$, we obtain (4. 22) $\sum_{\mu} \operatorname{Re} \int \phi_{\mu} A u \cdot \phi_{\mu} \bar{u} \frac{d x}{\rho} \geqq c_{1} \sum_{\mu, j} \int\left|\frac{\partial}{\partial x_{j}}\left(\widetilde{P}_{\mu} u\right)\right|^{2} \frac{d x}{\rho}-K_{1} \int \frac{|u|^{2}}{\rho} d x$.

Next consider $\int \varphi_{\lambda}^{*} A u \cdot \varphi_{\lambda}^{*} \bar{u} \frac{d x}{\rho}$. We write $A u=-\sum_{j, k \neq 0}^{n-1} \partial_{j}\left(a_{j k} \partial_{k} u\right)$ $+B u+\tau_{0}(x) \frac{\partial u}{\partial \nu}+\tilde{d}_{0}(x) \frac{u}{r}+\sum_{j=1}^{n-1} c_{j}(x) r^{-\beta} \partial_{j} u$. Since $B$ is a first order operator with respect to $\left(\partial_{0}, \cdots, \partial_{n-1}\right)$, we have

$$
\begin{aligned}
& \sum_{\lambda}\left|\int \varphi_{\lambda}^{*} B u \cdot \varphi_{\lambda}^{*} \bar{u} \frac{d x}{\rho}\right| \leqq \sum_{\lambda}\left|\int B\left(\varphi_{\lambda}^{*} u\right) \cdot \varphi_{\lambda}^{*} \bar{u} \frac{d x}{\rho}\right| \\
& \quad+\sum_{\lambda}\left|\int\left[\varphi_{\lambda}^{*}, B\right] u \varphi_{\lambda}^{*} \bar{u} \frac{d x}{\rho}\right| \leqq \eta \sum_{\lambda} \int \sum_{j=0}^{n-1}\left|\partial_{j}\left(\varphi_{\lambda}^{*} u\right)\right|^{2} \frac{d x}{\rho}+C(\eta) \\
& \quad \int|u|^{2} \frac{d x}{\rho} .
\end{aligned}
$$

On the other hand,

$$
\begin{gathered}
\int \varphi_{\mu}^{*} \tau_{0}(x) \frac{\partial u}{\partial \nu} \varphi_{\mu}^{*} \bar{u} \frac{d x}{\rho}=\int \tau_{0}(x) \frac{\partial}{\partial \nu}\left(\varphi_{\mu}^{*} u\right) \cdot \varphi_{\mu}^{*} \bar{u} \frac{d x}{\rho} \\
\quad+\int \tilde{c}_{0}(x)\left[\varphi_{\mu}^{*} \frac{\partial}{\partial \nu}\right] u \cdot \varphi_{\mu}^{*} \vec{u} \frac{d x}{\rho} .
\end{gathered}
$$


and $\left|\left[\varphi_{\mu}^{*}, \frac{\partial}{\partial \nu}\right] u\right| \leqq C \rho^{-1}(r)|u| \leqq C-\frac{|u|}{r}$, hence by virtue of Lemma.

4. 1, it holds.

$$
\text { (4. 24) } \begin{aligned}
& \sum_{\lambda}\left|\int \varphi_{\lambda}^{*} \bar{c}_{0}(x) \frac{\partial u}{\partial \nu} \cdot \varphi_{\lambda}^{*} \bar{u} \frac{d x}{\rho}\right| \leqq \eta \sum_{\lambda} \int_{j=0}^{n-1}\left|\partial_{j}\left(\varphi_{\lambda}^{*} u\right)\right|^{2} \frac{d x}{\rho}+C(\eta) \\
& \int|u|^{2} \frac{d x}{\rho}
\end{aligned}
$$

and similarly

(4. 25) $\quad \sum_{\lambda}\left|\int \varphi_{\lambda}^{*} \widetilde{d}_{0}(x) \frac{u}{r} \varphi_{\lambda}^{*} \bar{u} \frac{d x}{\rho}\right| \leqq$

$$
\leqq \eta \sum_{\lambda} \sum_{j=0}^{n-1} \int\left|\partial_{j}\left(\varphi_{\lambda}^{*} u\right)\right|^{2} \frac{d x}{\rho}+C(\eta) \int|u|^{2} \frac{d x}{\rho} .
$$

(4. 26) $\quad \sum_{\lambda}\left|\int \varphi_{\lambda}^{*} z_{k}(x) r^{-\beta} \partial_{k} u \varphi_{\lambda}^{*} \bar{u} \frac{d x}{\rho}\right| \leqq \eta \sum_{j=0}^{n-1} \int \mid\left(\left.\partial_{j}\left(\varphi_{\lambda}^{*} u\right)\right|^{2} \frac{d x}{\rho}\right.$

$$
+C(\eta) \int|u|^{2} \frac{d x}{\rho} \quad(1 \leqq k \leqq n)
$$

Finally we note $\int \phi_{\lambda}^{*}\left(-\sum_{j, k \rightarrow 0}^{n-1} \partial_{j}\left(\bar{a}_{j k} \partial_{k} u\right)\right) \varphi_{\lambda}^{*} \bar{u} \frac{d x}{\rho}$

$$
=-\int \sum_{j, k=0}^{n-1} \partial_{j}\left(\tilde{a}_{j k} \partial_{k}\left(\varphi_{\lambda}^{*} u\right)\right) \varphi_{\lambda}^{*} \bar{u} \frac{d x}{\rho}+\int\left[\varphi_{\lambda}^{*},-\sum_{j, k=0}^{n-1} \partial_{j}\left(a_{j k} \partial_{k}\right)\right] u \cdot \varphi_{\lambda}^{*} \bar{u} \frac{d x}{\rho}
$$

and we can see easily using the same technique as was applied to $\int\left[\tilde{\varphi}_{\mu}, A\right] u \cdot \tilde{p}_{\mu} \bar{u} \frac{d x}{\rho}$.

$$
\text { (4.27) } \begin{aligned}
\mid \int & {\left[\varphi_{\lambda}^{*},-\sum_{j, k=0}^{n-1} \partial_{j}\left(\tilde{a}_{j k} \partial_{k}\right)\right] u \cdot \varphi_{\lambda}^{*} \bar{u} \frac{d x}{\rho} \mid } \\
& \leqq \eta \sum_{j \sim s 0}^{n-1} \int\left|\partial_{j}\left(\varphi_{\lambda}^{*} u\right)\right|^{2} \frac{d x}{\rho}+C(\eta) \int|u|^{2} \frac{d x}{\rho} .
\end{aligned}
$$

By integration by parts after the local transformation (1.4), we get

$$
\text { (4. 28) } \begin{aligned}
- & -\sum_{j, k=0}^{n-1} \int \partial_{j}\left(\tilde{a}_{j k} \partial_{k}\left(\varphi_{\lambda}^{*} u\right)\right) \varphi_{\lambda}^{*} \bar{u} \frac{d x}{\rho} \\
& \geqq C \sum_{j, k=0}^{n-1} \int\left|\partial_{j}\left(\varphi_{\lambda}^{*} u\right)\right|^{2} \frac{d x}{\rho}-K_{2} \int|u|^{2}-\frac{d x}{\rho},
\end{aligned}
$$

and taking $\eta>0$ sufficiently small, we obtain (4.20). For the last 
On Dirichlet problems for some degenerate elliptic equations 393

part, for example, consider $u(x)=v \circ \theta^{-1}$. where $\theta$ denotes the local transformation (1.4) and $v(\nu, \tau)=\log (1+\phi(\nu)) u(\tau)\left(w(\tau) \in L^{*}(\tau)\right)$ near $\nu=0$ and of bounded support. It is easily seen $v(\nu, \tau)$ $\in W_{0}^{1}\left(R_{+}^{n}, \phi\right)$ but $\int_{R_{+}^{n}} \frac{\partial \nu}{\partial \nu} v \cdot v \frac{d \nu d \tau}{\rho}$ diverges. $\quad$ (Q. E. D.)

It is to be noted that the formal adjoint of $\partial / \partial x_{j}$ with respect to the inner product of $W^{\circ}(\Omega, \rho)$ becomes

$$
\left(\frac{\partial}{\partial x_{j}}\right)^{*}=-\frac{\partial}{\partial x_{j}}-\rho \frac{\partial \rho^{-1}}{\partial x_{j}}
$$

and $\rho \frac{\partial \rho^{-1}}{\partial x_{j}}$ behaves of most like $r^{-1}$ near $\Gamma$.

Definition 4. 1. $u(x) \in W_{0}^{1}(\Omega, \rho)(\alpha<1 / 2)$ is called to be a weak solution of (4.1), if $u(x)$ satisfies for all $v(x) \in W_{0}^{1}(\Omega, \rho)$

$$
\left\langle u,{\overline{A^{*} u}}_{\rangle_{\rho, \Omega}}=(f, v)_{\rho, Q} .\right.
$$

The following lemma is easy.

Lemma 4. 3. If $a_{j k}(x) \in \mathscr{B}^{3}\left(\Omega_{\delta}^{\mathfrak{c}}\right), b_{j}(x) \in \mathscr{B}^{1}\left(\Omega_{\xi}^{\prime}\right)$ and $c(x) \in \mathscr{B}^{0}\left(\Omega_{\xi}^{\prime}\right)$ with each $\delta>0$, and $\tilde{a}_{j k} \in \mathscr{B}^{3}\left(\omega_{\lambda}^{*}, \rho\right), \tilde{b}_{j}(x)$ and $\bar{c}_{j}(x) \in \mathscr{B}^{1}\left(\omega_{\lambda}^{*}, \rho\right)$ and $\tilde{d}_{\mathrm{o}}(x), \mathcal{d}(x) \in \mathscr{B}^{\circ}\left(\omega_{\lambda}^{*}, \rho\right)$ for each $\omega_{\lambda}^{*}$ in (4.2), then the coefficients of $A^{*}$ satisfy the assumptions in Proposition 4. 1 .

Now we are in a position to solve (4.1), that is, using the Gårding inequality for $A^{*}$ and applying the Lax-Milgram lemma we obtain

Theorem 4.1. If the coefficient of $A$ satisfy the assumptions in Lemma 4. 3, and $\lambda>0$ is sufficiently large, then there exists a unique weak solution $u(x) \in W_{0}^{1}(\Omega, \rho)$ of

$$
\left\{\begin{array}{l}
A u+\lambda u=f \\
\left.u\right|_{\Gamma}=0
\end{array}\right.
$$

for any $f(x) \in W_{0}^{1}(\Omega, \rho)^{\prime}$.

5. Differentiability theorem and alternative theorem of Fredholm. One of the main subjects in this section is to show that any weak solution of Dirichlet problem belongs to $W^{2}(\Omega, \rho)$ if $f(x) \in W^{\circ}(\Omega, \rho)$ and $\alpha<1 / 3$. Since our question is local, we 
shall transfer it to that in $R_{+}^{n}$ by the local transformation $\theta ; x \rightarrow(\nu, \tau)$ defined by (1.4).

We start with the following

Lemma 5. 1. (Poincaré). Let $u(x, y)$ be in $W_{0}^{1}\left(R_{+}^{n}, \phi\right)$ with bounded and fixedisupport, then we have

$$
\int_{R_{+}^{n}}|u(x, y)|^{2} d \phi d y \leqq C \int_{R_{+}^{n}}|D u|^{2} d \phi d y
$$

where $D$ denotes $D_{\rho}$ or $D_{y}$ and $C$ is a positive constant depending only on the diameter of the support of $u(x, y)$ and becomes small, when the diameter of supp. $u$ is small.

Let us denote the transformed operater by $A$ also ;

$$
\begin{aligned}
A & =-a_{00}(x, y)\left(\rho \frac{\partial}{\partial x}\right)^{2}-\sum_{k=1}^{n-1} a_{0, k}(x, y)\left(\rho \frac{\partial}{\partial x}\right)\left(\frac{\partial}{\partial y_{k}}\right) \\
& -\sum a_{j k}(x, y) \frac{\partial^{2}}{\partial y_{j}}+b_{0}(x, y)\left(\rho \frac{\partial}{\partial x}\right) \\
& +\sum_{j=1}^{n-1} b_{j}(x, y) \frac{\partial}{\partial y_{j}}+\sum_{j=1}^{n-1} x^{-\beta} c_{j}(x) \frac{\partial}{\partial y_{j}}+c_{0}(x, y) \frac{\partial}{\partial x} \\
& +d_{0}(x, y)+x^{-1} d(x, y) \quad(\text { near } x=0)(\beta<1-\alpha)
\end{aligned}
$$

where $a_{j k}(x, y) \in \mathscr{B}^{3}\left(R_{+}^{n}, \phi\right), b_{j}(x), c_{j}(x) \in \mathscr{B}^{1}\left(R_{+}^{n}, \phi\right)$ and $\lambda_{0}(x, y)$, $d(x, y) \in \mathscr{B}^{0}\left(R^{n}, \phi\right)$.

We denotes by $\sum_{8}$ the hemi-sphere of radius $\delta$;

$$
\sum_{\delta}=\left\{(x, y) ; x^{2}+|y|^{2}<\delta^{2}, x>0\right\} .
$$

To accomplish our aim, the following proposition is important.

Proposition 5. 1. Let $u(x, y)$ be in $W^{1}\left(R_{+}^{n}, \phi\right)$ and $\beta u \in$ $W_{0}^{1}\left(\sum_{8}, \phi\right)$ with $\alpha<1 / 3$ for any $\beta(x, y) \in C_{0}^{\infty}\left(B_{8}\right)$ (real valued) such that $\left(\rho \frac{\partial}{\partial x}\right)^{k} \beta(x, y)(k=0,1, \cdots)$ are continuous and bounded, where $B_{0}$ denotes the ball of radius $\delta$ with its centre at origin, and $u(x, y)$ satisfies

$$
A u=f(x, y),\left(f(x, y) \in W^{0}\left(R_{+}^{n}, \phi\right)\right),
$$

then if $\delta>0$ is sufficiently small, $u(x, y)$ belongs to $W^{2}\left(\sum_{\delta_{-}}, \phi\right)$ with $\varepsilon<\delta$. 
On Dirichlet problems for some degenerate elliptic equations 395

Proof. Since $u(x, y) \in W^{1}\left(R_{+}^{n}, \phi\right)$, we have by (5.3)

$$
\begin{aligned}
& -a_{00}(x, y)\left(\rho \frac{\partial}{\partial x}\right)^{2} u-\sum a_{0, k}(x, y)\left(\rho \cdot \frac{\partial}{\partial x}\right)\left(\frac{\partial}{\partial y_{k}}\right) u \\
& -\sum a_{j k}(x, z) \frac{\partial^{2} u}{\partial y_{j} \partial y_{k}}+\sum x^{-\beta} c_{j}(x, y) \frac{\partial u}{\partial y_{j}}+c_{0}(x, y) \frac{\partial u}{\partial x} \\
& +x^{-1} d(x, y) u=f(x, y)-b_{0} \rho \frac{\partial u}{\partial x}-\sum b_{j} \frac{\partial u}{\partial y_{j}} \\
& -d_{0} u \in W^{\circ}\left(R_{+}^{n}, \phi\right),
\end{aligned}
$$

and we may assume that $a_{00}(x, y) \equiv 1$. Multiplying $\beta(x, y)$ to both sides of (5.4) and noting $\left[\beta,\left(\rho \frac{\partial}{\partial x}\right)^{2}\right] u,\left[\beta, a_{0, k}\left(\rho \frac{\partial}{\partial x}\right)\left(\frac{\partial}{\partial x_{k}}\right)\right] u$, $\left[\beta, a_{j k} \frac{\partial^{2}}{\partial y_{j} y_{k}}\right] u,\left[\beta, x^{-\beta} c_{j} \frac{\partial}{\partial y_{j}}\right] u$, and $\left[\beta, c_{0} \frac{\partial}{\partial x}\right] u \in W^{0}\left(\sum_{\delta}, \phi\right)$, we obtain

$$
\begin{aligned}
& -\left(\rho \frac{\partial}{\partial x}\right)^{2}(\beta u)-\sum a_{0, k}\left(\rho \frac{\partial}{\partial x}\right)\left(\frac{\partial(\beta u)}{\partial y_{k}}\right)-a_{j k} \frac{\partial^{2}(\beta u)}{\partial y_{j} \partial y_{k}} \\
& +x^{-\beta} c_{j} \frac{\partial(\beta u)}{\partial y_{j}}+c_{0} \frac{\partial(\beta u)}{\partial x}+x^{-1} d(x, y) \beta u=g(x, y) \in W^{0}\left(\sum_{\delta}, \phi\right) .
\end{aligned}
$$

Now with the aid of the Gårding inequality and Lemma 5. 1., we have

$$
R e\langle A c, \overline{\mathcal{T}}\rangle \geqq c\|\varphi\|_{1, \phi} \quad\left(\varphi \in W_{0}^{1}\left(\sum_{8}, \phi\right)\right)
$$

hence

(5.5) $\quad c\|\varphi\|_{1,3} \leqq\|A \varphi\|_{-1, \phi}$

here $\|A \varphi\|_{-1, \phi}$ stands for the norm of $A u$ in $W_{0}^{\prime}\left(\Sigma_{\delta}, \phi\right)^{\prime}$, the dual space of $W_{0}^{1}\left(\sum_{\delta}, \phi\right)$. Thus by taking the difference quatient of tangential direction for $\varphi=\beta u$, we can see $\frac{\partial^{2}}{\partial y_{j} \partial y_{k}}(\beta u)$ and $\rho \frac{\partial^{3}}{\partial x} \frac{\partial y_{j}}{\beta u}(\beta u)$ belong to $W^{\mathrm{o}}\left(R_{+}^{\mathrm{n}}, \phi\right)$, hence we have

$$
\begin{aligned}
& -\left(\rho \frac{\partial}{\partial x}\right)^{2}(\beta u)+\frac{\partial}{\partial x}\left(c_{0} \beta u\right)-\frac{\partial c_{0}}{\partial x}(\beta u)+\sum x^{-\beta} \frac{\partial}{\partial y_{j}}\left(c_{j} \beta u\right) \\
& -\sum x^{-\beta} \frac{\partial c_{j}}{\partial y_{j}}(\beta u)+x^{-1} d(x, y)(\beta u)=h(x, y) \in W^{\circ}\left(\sum_{\delta}, \phi\right) .
\end{aligned}
$$


Since $\alpha<1 / 3, \beta<1-\alpha$ and $\left|\frac{\partial c_{0}}{\partial x}\right| \leqq K x^{-\infty}$ near $x=0$, we see $\frac{\partial c_{0}}{\partial x}(\beta u)$ and $\Sigma x^{-\beta} \frac{\partial c_{j}}{\partial y_{j}}(\beta u) \in W^{o}\left(\Sigma_{\delta}, \phi\right)$, here we used the fact

$$
|\psi| \leqq x^{1-\infty / 2} \psi_{1}(y), \phi_{1}(y) \in L^{2}\left(R^{n-1}\right) \quad\left(\vee \psi \in W_{0}^{1}\left(R_{+}^{n}, \phi\right)\right)
$$

Next consider $\int_{x}^{\delta} \frac{\partial}{\partial x}\left(c_{0} \beta u\right) \frac{d x}{\rho}$ and $\int_{x}^{\delta} \frac{d(x, y)}{x}(\beta u) \frac{d x}{\rho}$. Using

(5. 7) $\quad \int_{x}^{\delta} \frac{\partial}{\partial x}\left(c_{0} \beta u\right) \frac{d x}{\rho} \leqq \int_{0}^{\delta}\left|D_{\rho}\left(c_{0} \beta u\right)\right|^{2} d \phi \int_{x}^{\delta} \frac{d x}{\rho^{3}}$

$$
\int_{x}^{8}\left|\frac{d(x, y)}{x} \beta u\right| \frac{d x}{\rho} \leqq \psi_{2}(y) \int_{x}^{8} \frac{1-\alpha}{x^{2}} \frac{d x}{x \rho} \quad \psi_{2}(y) \in L^{2}\left(R^{n-1}\right)
$$

and by virtue of $\alpha<1 / 3$, we see the left hands of (5.7) and (5.8) are majorized by some $\psi_{3}(y) \in L^{2}\left(R^{n-1}\right)$. This shows that $\rho \frac{\partial}{\partial x}(\beta u)$ is also majorized by some $L^{2}\left(R^{n-1}\right)$ function, therefore we have (5. 9) $\quad|\beta u| \leqq x^{1-\infty} \psi_{3}(y), \psi_{3}(y) \in L^{2}\left(R^{n-1}\right)$.

Thus we obtain

$$
\begin{aligned}
& \text { (5. 10) }\left|\frac{\partial}{\partial x}(\beta u)\right| \leqq x^{-\star} \psi_{s}(y) \\
& \text { (5. 11) }\left|\frac{\beta u}{x}\right| \leqq x^{-\star} \psi_{\mathrm{s}}(y)
\end{aligned}
$$

with $\psi_{1}(y)$ and $\psi_{5}(y) \in L^{2}\left(R^{n-1}\right)$, and this shows $\frac{\partial}{\partial x}(\beta u)$ and $x^{-1} \beta u \in W^{0}\left(\sum_{8}, \phi\right)$ and finally we see $\left(\rho \frac{\partial}{\partial x}\right)^{2}(\beta u) \in W^{0}\left(\sum_{8}, \phi\right)$. This completes the proof.

Remark. $\beta(x, y)$ used above is constructed as follows: let $q(t)$ be a $C_{0}^{\infty}\left(R_{+}^{1}\right)$ function such that

i) $0 \leqq q(t) \leqq 1$.

ii) $q(t)=1$ if $\quad-\delta^{\prime} \leq t \leq \delta^{\prime} \quad\left(0<\delta^{\prime}<\delta\right)$

iii) $q(t)=0$ if $|t| \geqq \delta$,

then $\beta(x, y)=q(\tilde{\phi}(x)) \prod_{j=1}^{n-1} q\left(y_{j}\right)$ is our demanded one. 
On Dirichlet problems for some degenerate elliptic equations 397

By the above proposition we can prove

Theorem 5.1. If $f(x)$ is in $W^{\circ}(\Omega, \rho)$, then the weak solution of Dirichlet problem belongs to $W^{2}(\Omega, \rho)$. Here we assume $\alpha<1 / 3$ and this is the best possible condition on $\alpha$.

There will arise naturally the question whether $f(x)$ is given in $W^{s}(\Omega, \rho)$, then the solution $u(x)$ belongs to $W^{s=2}(\Omega, \rho)$ or not. The answer to this question is negative in general, but if the term involving $\partial / \partial \nu$ does not occur, and $c_{j}=0(j=1,2, \cdots, n-1)$ and $d_{0}(x, y)=0$, then the answer becomes positive.

Our next subject in this section is to establish the alternative theorem of Fredholm for our problem.

Lemma 5. 2. Let us denote by $K_{0}^{1}\left(\sum_{\delta}, \phi\right)$

$$
\left\{u(x, y) \in W_{0}^{1}\left(R_{+}^{n}, \phi\right) ;\|u\|_{1, w} \leqq 1 \text {, and Supp } u \subset \sum_{d}\right\} \text {, }
$$

then $K_{0}^{1}\left(\Sigma_{\delta}, \phi\right)$ is compact in $W^{0}\left(R_{-}^{n}, \phi\right)$.

Proof. Let us denote by $\tilde{u}(x, y)$ the extention of $u(x, y)$ to $R^{n}$ by setting $\tilde{u}(x, y)=0$ for $x<0$.

First note that for ${ }^{v} u \in W_{0}^{1}\left(R_{+}^{n}, \phi\right)$

$$
\tilde{\rho}(x) \frac{\partial \ddot{u}}{\partial x}=\widetilde{\rho(x) \frac{u}{x}}
$$

and set

$$
\varphi(\xi, \eta)=\int_{R^{n}} u(x, y) e^{-2 x i \xi \tilde{\xi}(x)-z x_{i} \eta \cdot y} d \tilde{\phi} d y
$$

for $u(x, y) \in K_{0}^{1}\left(\sum_{\delta}, \phi\right)$. Then since $\tilde{u}=u$ when $x>0$, we have

$$
\begin{aligned}
& u(x, y)=\int_{|\xi|+i \eta \leqslant \leqslant R} e^{2 z i \xi \bar{\phi}(x) \cdots 2 \times i \eta \cdot y} \varphi(\xi, \eta) d \xi d \eta \\
& \div \int_{\xi i-i n i \gtrless R} e^{2 \pi i \xi \bar{\eta}(x)+2 \pi i \eta \eta y} \varphi(\xi, \eta) d \xi d \eta \\
& \equiv u_{1}(x, y)+u_{2}(x, y) \text {. }
\end{aligned}
$$

Thus by Plancherel's theorem, it holds

$$
\left.|| u_{2}\right|_{0 . \tilde{\phi}} \leqq \frac{1}{2 \pi R} \int\left(1+4 \pi^{2}\left(\xi^{2}+|\eta|^{2}\right)\right)|\varphi(\xi, \eta)|^{2} d \xi d \eta \leqq \frac{\text { const. }}{R}
$$

and we see that $\left\{u_{1} ; u \in K_{0}^{1}\left(\sum_{\delta}, \phi\right)\right\}$ makes an equi-continuous 
and uniformly bounded set in $\sum_{8}$. Hence by Ascoli-Arzela's theorem, we can find a sequence in $\left\{u_{1} ; u \in K_{0}^{1}\left(\sum_{b}, \phi\right)\right\}$ which converges uniformly in $\sum_{\delta}$, and since $\rho^{-1}$ is integrable in $(0, \delta)$, we see this sequence converges in $W^{\circ}\left(\sum_{\delta}, \phi\right)$. Now according to $R=1,2, \cdots$, using the diagonal method we can select a sequence $\left\{u_{j}\right\}$ in $K_{0}^{1}\left(\sum_{\delta}, \phi\right)$ which converges in $W^{0}\left(\sum_{\delta}, \phi\right)$.

For the general bounded domain, by taking a finite partition of unity of class $\mathscr{B}^{\infty}(\Omega, \rho)$ and using the local transformation defined by (1.4), we can state

Proposition 5.2. Let $\Omega$ be a bounted domain, then the bounded set of $W_{0}^{1}(\Omega, \rho)$ is precompact in $W^{\circ}(\Omega, \rho)$.

Thus by Riesz-Schauder's theory, we have

Theorem 5.2. Let us consider the following equations in a bouneed domain

$$
\text { (5.16) } \quad\left(A^{*}+\bar{\lambda}\right) v=\left.g \quad v\right|_{\Gamma}=0,
$$

then

1) the eigenvalues of $-A$ in $(5.15)$ make a sequence accumulating at most at infinity and the eigenvalues of $-A^{*}$ are given by the complex conjugates of them.

2) let $\lambda_{2}$, be an eigenvalue of $-A$, then the eigen space correspoding to $\lambda_{2}$ is of finite dimensions and its dimension coincides with that of the eigenspace of $-A^{*}$ corresponding to $\bar{\lambda}_{2}$,

3) when $\lambda \notin\left\{\lambda_{,}\right\},(5.15)$ has a uniques solution $u(x) \in W_{0}^{1}(\Omega, \rho)$ with $\alpha<\frac{1}{2}$ (resp. $u(x) \in W^{2}(\Omega, \rho) \cap W_{0}^{1}(\Omega, \rho)$ with $\alpha>1 / 3$ for any $f \in W^{\circ}(\Omega, \rho)$ and the mapping: $f \rightarrow u$ is bounded in $W^{\circ}(\Omega, \rho)$. And for (5.16) we have the same result.

4) if $\lambda=\lambda$, a necessary and sufficient condition in order that (5.15) has a solution is that $f(x)$ is orthogonal to the eigenspace corresponding to $\bar{\lambda}_{,}$, the eigenvalue of $-A^{*}$ in (5.18).

6. Dirich let problems for higher order equations. For the Dirichlet problems for the higher order equations, we can have 
the analogous results to the second order cases.

Consider a differential operator of order $2 m$ in $\Omega$ which can be written near as

(6. 1) $\quad A(x, D)=\sum_{|\mu| \leqslant 2 m} \hat{a}_{\mu}(x) \partial^{\mu}+$ lower order operator, where

$$
\begin{aligned}
& \partial=\left(\left(\rho(r) \frac{\partial}{\partial \nu}, \frac{\partial}{\partial \tau_{1}}, \cdots, \frac{\partial}{\partial \tau_{n-1}}\right)\right. \text { and it is assumed that } \\
& A(x, D) \text { is normalized by the requirement }
\end{aligned}
$$

(6. 2) $(-1)^{m} \operatorname{Re} \sum_{|\mu|=2 m} \tilde{a}_{\mu}(x) \xi^{\mu} \geqq c|\xi|^{2 m} \quad(c$ is independent of $x$ ).

Our equation is

$$
\left\{\begin{array}{l}
A u=f \in W^{\circ}(\Omega, \rho) \\
\left.\left(\rho(r) \frac{\partial}{\partial \nu}\right)^{j} u\right|_{\Gamma}=0 \quad(j=0, \cdots, m-1) .
\end{array}\right.
$$

Definition 6. 1. $u(x) \in W_{0}^{m}(\Omega, \rho)$ is said to be a weak solution of (6.3), if $u(x)$ satisfies for any $v(x) \in W_{0}^{m}(\Omega, \rho)$

(6. 4) $\left\langle u, \overline{A^{*} v}\right\rangle_{\mathrm{p}, \mathrm{Q}}=(f, v)_{\mathrm{p}, \mathrm{Q}}$.

Corresponding to Lemma 4.1., the following lemma holds.

Lemma 6.1. Let $B(x, D)$ be a differential operator which can be uritten near $\Gamma$ as

$$
\begin{aligned}
& B(x, D)=\sum_{i=1 k \leq \sum^{2 m-1}-1} \tilde{b}_{j,(k)}(x)\left(\rho(r) \frac{\partial}{\partial \nu}\right)^{j}\left(\frac{\partial}{\partial \tau}\right)^{k} \\
& +\sum_{i-j+\ell-|k| \leqq 2 m-1} \tau_{i, j, k), l}(x)\left(\rho(r) \frac{\partial}{\partial \nu}\right)^{i}\left(\frac{\partial}{\partial \nu}\right)^{j} r^{-l+1-\beta}\left(\frac{\partial}{\partial \tau}\right)^{k} \\
& +\tilde{d}(x) \quad(\beta<1-\alpha)
\end{aligned}
$$

where $\tilde{b}_{j,(\omega)}(x) \in \mathscr{B}^{3 m-j-|k|-1}(\omega, \rho), c_{i, j,(k), \gamma}(x) \in \mathscr{B}^{z m-i-j-|k|-1}(\omega, \rho)$ and $d(x) \in C^{\circ}(\omega)$ with $|d(x)| \leqq K r^{-3 m+1}$ for each boundary patch $\omega$, then we have for any $u(x) \in W_{0}^{m}(\Omega, \rho)$

$$
\begin{aligned}
& \int_{\alpha}|B u \cdot \bar{u}| \frac{d x}{\rho} \leqq \varepsilon\|u\|_{m, \rho, \alpha}^{2}+C(\varepsilon)\|u\|_{0, \rho, \Omega}^{2} \\
& \int_{\mathbf{a}}\left|B^{*} u \cdot \bar{u}\right| \frac{d x}{\rho} \leqq \varepsilon|| u\left\|_{m, \rho, \alpha}^{2}+C(\varepsilon)\right\| u \|_{0, \rho, \alpha}^{2}
\end{aligned}
$$

if $\alpha<1 / 2 m$, here $B^{*}$ denotes the formal adjoint of $B$ in $W^{\circ}(\Omega, \rho)$. 
Finally as for the coefficients of $B$, we assume that they are all in $C^{3 m-1}(\Omega)$.

Remark. The condition on $\alpha$ is the best possible one unless $\bar{c}_{0,2 m-1,(0), 0}(x)$ is identically zero and $|d(x)| \leqq K r^{-p}$ with $p<2 m-1$.

By virtue of Lemma 6.1., we have the Gårding inequality for higher order case.

Proposition 6. 1. Suppose $\bar{a}_{\mu}(x)$ in (6. 1$)$ are all in $\mathcal{B}^{3 m}(\omega, \rho)$, coefficients of $A(x, D)$ are all in $C^{3 m}(\Omega)$, and the lower order term which appears in (6.1) is of same type as $B(x, D)$ in Lemma 6.1., then we have positive constants $C$ and $K$ such that

$$
R e\left\langle u, \overline{A^{*} u}\right\rangle_{\rho, u} \geqq C\|u\|_{m, \rho, u}^{2}-K\|u\|_{0, \rho, Q}^{2}
$$

for all $u(x) \in W_{0}^{m}(\Omega, \rho)$ with $\alpha<1 / 2 m$.

Proof. Taking account of Lemm 6.1. if suffices to show (6.7) when $A^{*}=\sum_{|\mu| \rightarrow 3^{m}, a^{*}} \hat{a}^{*}(x) \partial^{\mu}$ with $a^{*}(x) \in \mathscr{B}^{m}(\omega, \rho)$. Further using the transformation (1.4), we can reduce our problem to the case in a half-space, that is $A^{*}=\sum_{\mu=2 m} a_{\mu}(x, y) \partial^{\mu}$ with $a_{\mu} \in \mathscr{B}^{m}\left(R_{+}^{n}, \rho\right)$ where $\partial=\left(\rho(x) \frac{\partial}{\partial x} \frac{\partial}{\partial y_{1}}, \cdots, \frac{\partial}{\partial y_{n-1}}\right)$.

Now let $u(x, y)$ be in $C_{0}^{\infty}\left(\sum_{8}\right)$. We regard $u(x, y)$ as an element of $C_{0}^{\infty}\left(R^{n}\right)$ by extending it identically zero for $x<0$. First we assume all $a_{\mu}$ to be constant, then using the weighted Fourier transformation (2.5) and Lemma 2.2., we have

$$
\operatorname{Re}\left\langle a, \overline{A^{*} u}\right\rangle_{s}=\operatorname{Re}\left(\mathcal{F}_{\bar{\rho}} u, \sum a_{\mu} \xi^{\mu} \mathscr{T}_{\tilde{\rho}} u\right) \geqq c\|u\|_{m, \mathrm{~b}}^{2}
$$

if $\delta>0$ is sufficiently small, thus since by the continuity of $a_{\mu}(x, y)$ and by the integration by parts, we have

$$
\left|\int\left(\sum_{\mu} a_{\mu}(x, y)-a_{\mu}(\delta, \vec{\delta})\right) \partial^{\mu} u, \bar{u} \frac{d x}{\rho}\right| \leqq \varepsilon(\delta)|| u\left|\left\|_{, p}+C(\delta)|| u\right\|_{0, p}^{z}\right.
$$

where $\delta=\frac{n-1}{(\delta, \cdots, \delta)}$, we can prove (6.7).

Using above proposition and applying the Lax-Milgram lemma, we obtain 
On Dirichlet problems for some degenerate elliptic equations 401

Theorem 6.1. Under the same assumptions on $A(x . D)$ as in Proposition 6. 1., there exists a unique solution of $(A+\lambda) u=f$ for any $f \in W_{0}^{m}(\Omega, \rho)^{\prime}$, if $\lambda>0$ is large cnough.

If we expect the weak solution be in $W^{2 m}(\Omega, \rho)$, we must impose more restrictions on the lower order operator and on $\alpha$. In fact we obtain

Theorem 6.2. Let $B(x, D)$ be the lower order operator which can be expressed near $\Gamma$ as

$$
\begin{aligned}
& B(x, D)=\sum_{i+1, i \leq 2 m+1} \tilde{b}_{j, j}(x)\left(\rho-\frac{\partial}{\partial \nu}\right)^{j}\left(\begin{array}{c}
\partial \\
\partial \tau
\end{array}\right)^{k} \\
& +\sum_{i, j,|x|, \imath \leq m} \tilde{c}_{i, j,(k), l}(x)\left(\rho \frac{\partial}{\partial \nu}\right)^{i}\left(-\frac{\partial}{\partial \nu}\right)^{j} r^{-i+1-\beta}\left(\frac{\partial}{\partial \tau}\right)^{k} d(x)
\end{aligned}
$$

with $|\tilde{d}(x)| \leqq K r^{-m}$, then the weak solution of (6.3) belongs to $W^{2 m}(\Omega, \rho)$, if $f(x) \in W^{\circ}(\Omega, \rho)$ and $\alpha<1 / 2 m+1$, and the condition on $\alpha$ is the best possible one.

Finally in $\Omega$ is a bounded domain, we can also apply the alternative theorem of Fredholm.

\section{RITSUMEIKAN UNIVERSITY}

\section{REFERENCES}

[1] M.S. Baouendi: Sur une class d'operateurs elliptiques dégénérès. Thèse Paris, 1966 (Bull. Soc. Math. France, 95, (1967), 45

[2] S. Mizohata: Theory of Partial Differential Equations. Iwanami Tokyo. 1966 (in Japanese).

[3] M.R.K. Murthy-G. Stampacchia: Boundary value probleme for some degenerate alliptic operators. Anuali di Math. pure et appl. (4) vol. LXXX, (1968), 1-122.

[4] A. Nakaoka: Mixed problems for degenerate hyperbalic equations of second order. Proc. Japan Acad. vol. 15, (1969), 706-709.

[5] A. Nakaoka: Boundary value problems for some degenerate elliptic equations of second order with Dirichlet condition. Proc. Japan Acad. vol. 46 (1970), 248-252.

[6] L. Nirenberg: Remarks on strongly elliptic partial differential equations. Comm. Pure Appl. Math. vol. 8, (1955), 648-674.

[7] N. Shimakura: Problèmes aux limites généraux du type elliptique dégénéré. to appear in J. Math. Kyoto Univ. 